\title{
Wireless Sensor Network Coverage Optimization Based on Fruit Fly Algorithm
}

\author{
https://doi.org/10.3991/ijoe.v14i06.8698 \\ Ren Song( $\left.{ }^{\bowtie}\right)$, Zhichao Xu, Yang Liu \\ Jilin University of Finance and Economics, Jilin, China \\ rensong1579@163.com
}

\begin{abstract}
To solve the defect of traditional node deployment strategy, the improved fruit fly algorithm was combined with wireless sensor network. The optimization of network coverage was implemented. Based on a new type of intelligent algorithm, the change step of fruit fly optimization algorithm (CSFOA) was proposed. At the same time, the mathematical modeling of two network models was carried out respectively. The grid coverage model was used. The network coverage and redundancy were transformed into corresponding mathematical variables by means of grid partition. Among them, the maximum effective radius of sensor nodes was fixed in mobile node wireless sensor network. The location of nodes was randomly cast. The location of sensor nodes was placed in fixed position nodes. The effective radius of nodes can be changed dynamically. Finally, combined with the corresponding network model, the improved algorithm was applied to wireless sensor network. The combination of the optimal solution of the node position and the perceptual radius was found through the algorithm. The maximum network coverage was achieved. The two models were simulated and verified. The results showed that the improved algorithm was effective and superior to the coverage optimization of wireless sensor networks.
\end{abstract}

Keywords-wireless sensor networks; coverage optimization; sensor nodes

\section{Introduction}

In recent years, the Internet of Things, cloud computing and big data have developed rapidly. The Internet of things is the key technology of the information interaction between the future reality and the virtual. It is also the key research direction to realize the "information society". The Internet of Things mainly has three communication sensing technologies, namely, intelligent sensing, identification technology and pervasive computing. Internet of Things technology is widely used in information processing, in order to achieve a complete integration of the physical world and the information world. As a core technology of Internet of Things, Wireless Sensor Network (WSN) is attracting much attention. The wireless sensor has three functions, such as information collection, processing and transmission. They correspond to the three modern basic information technologies, namely, sensor technology, computer 
technology and communication technology. They constitute the "sensory", "brain", and "nerve" of the information technology system.

As a new technology, wireless sensor network is widely used in military field, agricultural production, ecological monitoring and disaster warning, infrastructure status detection, industrial field, intelligent transportation, medical system and smart home. However, because the working environment of WSN is so bad that most of them can't deploy sensor nodes in the field, they can only deploy nodes in large-scale random delivery mode, which causes many urgent problems. For example, the acquisition of wireless sensor networks, processing and transmission of high-quality signals and the construction of sensor node hardware platform need to be studied. One of the key problems of sensor networks is the deployment optimization of nodes. The improvement of network coverage, the reduction of network energy consumption and the simplification of network model are the basis of the development of wireless sensor technology.

\section{$2 \quad$ Literature review}

Cheng and others as stated in [1] designed a delay-aware data collection network structure for wireless sensor networks. In addition, Sun and others proposed as stated in [2] a cooperative communication for wireless ad hoc and sensor networks. Wireless sensor network is a distributed sensor wireless ad hoc network. The end of the WSN node can perceive and inspect the sensors of the external world.

$\mathrm{Li}$ and Lin as stated in [3] designed a smart home control system based on wireless sensor networks. Luo and others as stated in [4] applied the opportunistic routing algorithm for relay node se-lection in wireless sensor networks. Rezvani and others as stated in [5] studied the data aggregation technique for wireless sensor networks. Srbinovska and others, as stated in [6], proposed environmental parameters monitoring, which used wireless sensor networks. Shu and others as stated in [7] optimized the transmit power-time and bit energy efficiency based on cdma wireless sensor networks. Younis and others as stated in [8] discussed the node clustering in wireless sensor networks. Yao and others as stated in [9] proposed an energy-efficient and lifetime-balancing data collection protocol for wireless sensor networks. Zhao and others as stated in [10] designed a mobile data gathering with load balanced clustering and dual data uploading in wireless sensor networks.

By deploying sensors, the information in the monitoring area is collected in real time and processed with a processing unit. Then, these data are sent to the remote network control center. The location of the node can be fixed and removable, and the sensor nodes can be wired or wirelessly connected to the Internet. Wireless sensor networks (WSN) are composed of many sensor nodes deployed in the monitoring area. There are three main elements in the network: sensor, perceiving object and observer structure.

In summary, the existing research mainly stays in the range of sensor coverage, but the problem of path optimization and localization precision has not been further involved. In view of network coverage optimization, the change step of fruit fly optimi- 
zation algorithm is designed based on the fruit fly algorithm. This method makes up for the shortcomings of current research on network coverage. It is noted that the new method is effective and superior to the coverage optimization of wireless sensor networks.

\section{Methodology}

\subsection{Wireless sensor network}

Wireless sensor network is a distributed sensor wireless ad hoc network. The end of the WSN node can perceive and inspect the sensors of the external world. By deploying sensors, the information in the monitoring area is collected in real time and processed with a processing unit. Then, these data are sent to the remote network control center. The location of the node can be fixed and removable, and the sensor nodes can be wired or wirelessly connected to the Internet. Wireless sensor networks (WSN) are composed of a large number of sensor nodes deployed in the monitoring area. There are three main elements in the network: sensor, perceiving object and observer structure. By perceiving, collecting, and processing the information of the perceiving object, it is finally sent to the observer. The structure of the wireless sensor is shown in Figure 1.

Cloud
compution $\leftrightarrow \begin{gathered}\text { Integration } \\ \text { node }\end{gathered} \leftrightarrow \stackrel{\begin{array}{c}\text { Internet or } \\ \text { satellite }\end{array} \leftrightarrow 4 \begin{array}{c}\text { Management } \\ \text { node }\end{array}}{4}$

Fig. 1. Structure diagram of wireless sensor network

In different applications, the composition of the sensor nodes is different. However, they are usually made up of 4 parts: the perception module, the communication module, the processor module and the energy supply module. The structure of the node function of the wireless sensor network is shown in Figure 2.

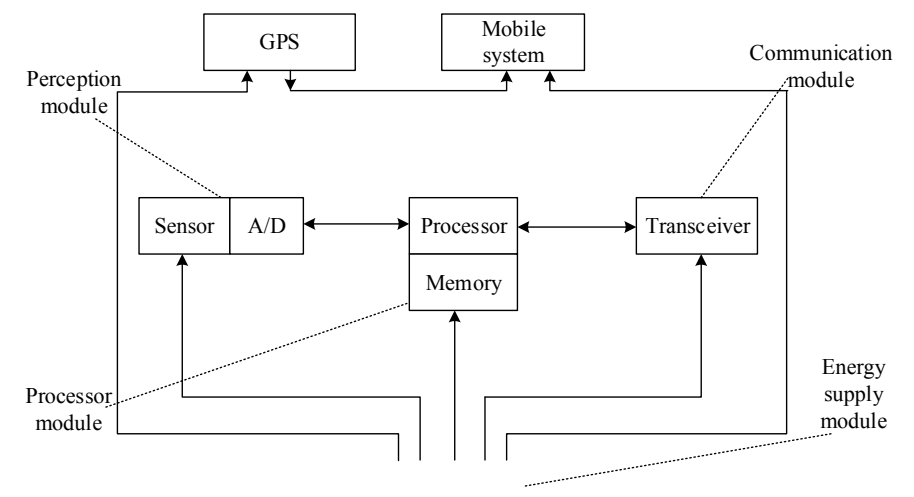

Fig. 2. The structure of the node function of the wireless sensor network 
From the horizontal direction, each part is the management unit of the network. From the vertical direction, each part is composed of the communication protocol layer of the network. According to the difference of function, the communication protocol layer can be divided into five layers: physical layer, data link layer, network layer, transmission layer and application layer. The network management unit is divided into three aspects according to its function: energy management, mobility management and task management. The network system structure of sensor communication is shown in Figure 3.

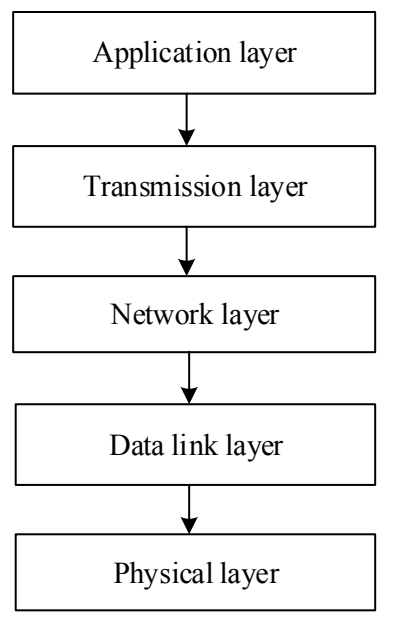

Fig. 3. The network system structure of sensor communication

Large scale: A large number of accurate data are collected in the monitoring target area, so the wireless sensor network will deploy sensor nodes on a large scale. The scale of WSN is reflected in two parts: one is the large target monitoring area of WSN; the other is the high density of sensor nodes in the target monitoring area. Wireless sensor networks (WSN) use a large number of sensor nodes to cooperate to improve the network communication and the quality of service.

Self-organization: In the use of wireless sensor networks, the location of the initial sensor nodes is generally not accurate. The structure between adjacent nodes is also uncertain. Therefore, the self-organizing ability of the sensor nodes is very important. It can automatically set and control the network. In addition, the cover void caused by the failure node needs to be remedied to increase the signal precision in the monitoring area. The topology of the network will also change. The self - organizing ability of the network ensures that the structure can cope with these changes.

Dynamic: The deployment of wireless sensor network is unpredictable, such as sensor node failure or scrapping, communication link structure change, WSN three elements (sensor, perceiving object and observer) location change, new node joining. This requires that the wireless sensor network can restructure the network topology in time and realize the dynamic change of the network. 
Coverage performance: The coverage performance of wireless sensor networks is related to the distribution mode and number of sensor nodes and the energy consumption of the nodes. The implementation of target monitoring area coverage, the optimal node distribution mode selection, the optimal number of nodes and the reduction of node energy consumption are the key to the research of wireless sensor network coverage performance.

Connectivity performance: In wireless sensor networks, the working ability of a single node is very small, and the performance of the perception range and network communication is very poor. On the premise of satisfying perception and coverage, wireless sensor networks should also guarantee the connectivity of wireless sensor networks. Connectivity and coverage are the basis for the normal operation of wireless sensor networks.

The life time of the network: The rated power of wireless sensor nodes is usually not high. The working environment of the node is usually very bad, and it is difficult to maintain the node. Therefore, the survival of a single node will affect the smooth operation of the entire network. By improving the energy utilization of each node, the whole network life time can be extended effectively.

Energy balance: Energy consumption is one of the most influential factors for the design and implementation of wireless sensor networks. The energy consumption of each node is different because of the different working modes of each node. Some nodes with higher frequency can be easily damaged, which makes the network coverage and the quality of work rapidly decline. Therefore, it is a key problem to ensure the energy balance of each node.

Fault tolerance and adaptability: The working environment of wireless sensor networks is generally very bad. It is often infeasible to maintain or replace a node. Therefore, the network needs strong fault tolerance and self-adaptability. It can automatically manage and repair the network through automatic adjustment, and improve the quality of service of the network.

The complexity of the algorithm: The wireless sensor network node is a small embedded system module. The computing power and storage space are very limited. The complexity of the coverage control algorithm must be taken into account. Too complex algorithm will bring the network slow speed, storage space and other disadvantages. Therefore, the complexity of the algorithm is also a key problem that must be considered when designing the network.

\subsection{Fruit fly algorithm}

The intelligent optimization algorithm has the global optimization performance. It is suitable for parallel processing. The algorithm generally has a strict theoretical basis. In theory, the optimal solution or approximate optimal solution can be found in a certain time. Optimization algorithm has many calculation modes, such as genetic algorithm, fruit fly algorithm, particle swarm algorithm, leapfrog algorithm, firefly algorithm and so on. In solving complex problems, a variety of computing modes can be mixed to maximize computing power. 
Fruit fly optimization algorithm (FOA) is a new type of intelligent optimization algorithm researched and developed by Pan Wenchao in 2011. It has good global optimization performance and can solve many optimization problems. Fruit fly has sensory awareness superior to other organisms, especially visual and olfactory. Depending on the sense of smell, fruit flies are well aware of various odorous molecules in the air and can even smell food tens of kilometers away. At the same time, with keen eyesight, it gathers with other fruit flies. Fruit fly algorithm is to imitate the foraging process of fruit flies and put forward a new type of intelligent optimization algorithm.

The fruit fly algorithm is less computationally intensive, the running time is short, the algorithm complexity is low, and the optimization precision is high. However, the time of the fruit fly algorithm is short, the algorithm theory and method are not mature, so the algorithm has a lot of defects. The stability of the fruit fly algorithm is not high, and it is very easy to fall into the local optimal. In the later period, the convergence precision is reduced and the convergence speed is slowed down. These are the contents and improvements that need to be studied when using the fruit fly algorithm.

\subsection{Change the Step of FOA}

According to the introduction of the fruit fly algorithm in the last section, it can be seen that the fruit fly algorithm has many advantages over other swarm intelligence algorithms. However, the fruit fly algorithm also has a large defect. The fruit fly individual iterates from the same starting point each time in the fruit fly algorithm. Then, the search is carried out in the random direction, and the random number in the fixed interval $[-H, H]$ is searched. When $H$ is large, the algorithm has strong global search ability and fast convergence speed, but the local search ability of the algorithm is low, and the convergence accuracy is not enough. On the contrary, if the $H$ is smaller, the local search ability is stronger. The global search capability is low. It leads to the slow convergence of the algorithm. Moreover, the algorithm is searched in the area, and the convergence result is easy to fall into the local optimal error solution centralization. In view of the above limitations, the change step of fruit fly optimization algorithm (CSFOA) proposed in this paper divides the search into several cycles. If 50 iterations are taken to a period of $T$, the step length in each cycle is changed in the form of $\operatorname{Sin}(x)$, which is as follows:

$$
\begin{aligned}
& X_{i}=X_{-} \alpha x i s+(2 \times \operatorname{rand}()-1) \times H_{i} \\
& \alpha=\bmod (i, T) \quad i \in[1, \text { Maxgen }] \\
& H_{i}= \begin{cases}L \times(\sin (i)+1)^{\alpha} \quad H_{i} \leq L \\
L & H_{i}>L\end{cases}
\end{aligned}
$$

Among them, $L$ is the algorithm for searching interval length, $T$ is the number of iterations of unit cycle, and $\bmod (i, T)$ is the rest of $i$ iteration relative to $T$. As can be seen from the formula (1), CSFOA first divides the whole search process into sev- 
eral cycles, which can increase the diversity of the search process. The algorithm can effectively jump out of local convergence and greatly reduce the possibility of local convergence. Then, CSFOA uses the $\operatorname{Sin}(x)$ function within each cycle to make the step change in the unit cycle $T$. When $\operatorname{Sin}(x)$ is increasing monotonously, the step length exponentially increases. The algorithm has a strong global search ability and can achieve fast convergence. The convergence result is not easy to fall into the local optimal. At the same time, the increase of the step length can solve the possible local convergence problem of $\operatorname{Sin}(x)$ in the last monotone decreasing interval. In the $\operatorname{Sin}(x)$ monotonically decreasing interval, the step length exponential type decreases, which enables the algorithm to complete a high precision search in a small range. The result has better convergence effect. The CSFOA process is shown in Figure 4.

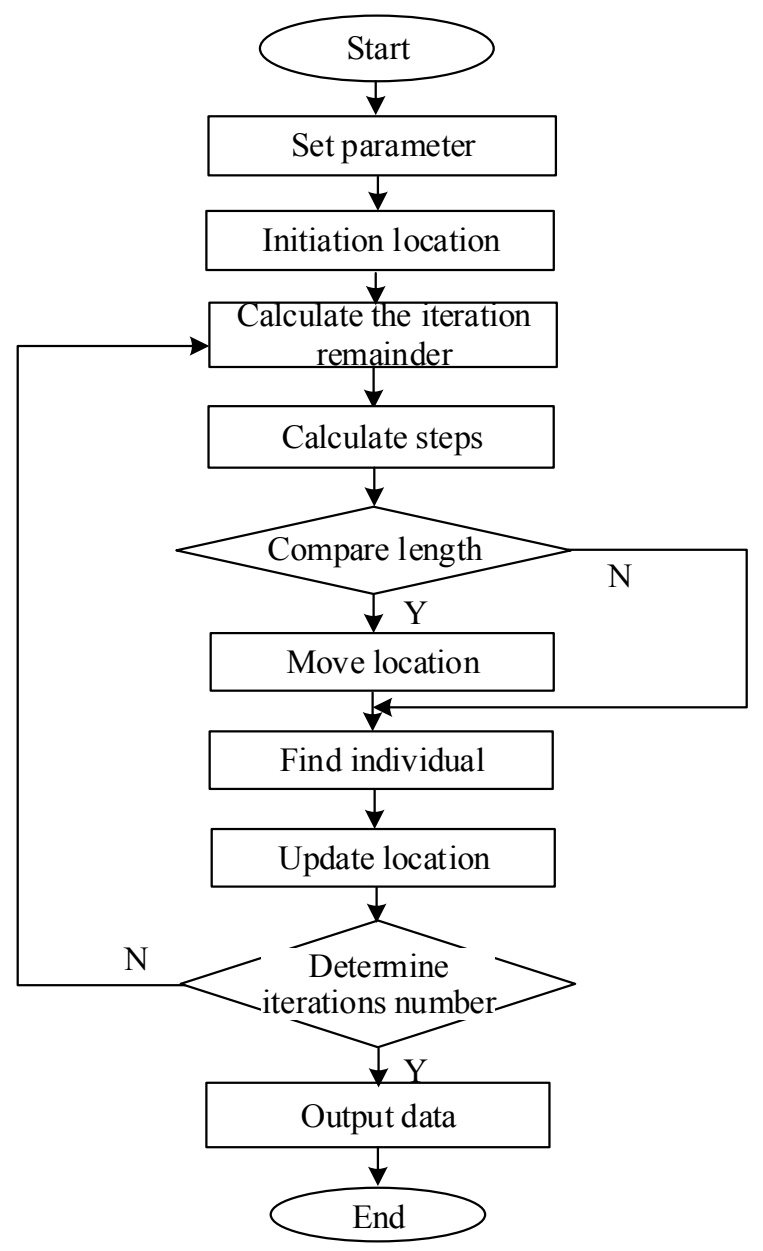

Fig. 4. The CSFOA process 


\section{$4 \quad$ Result Analysis and Discussion}

\subsection{Node model of wireless sensor network}

The grid coverage model is used. In a two-dimensional region of $a^{*} b$, the region is dispersed into $a^{*} b$ receiving point, and the location of each receiving point is expressed as $\left(a_{i}, b_{i}\right) . N$ sensor nodes are randomly deployed in the target area, and the rated effective radius of the node is R. Each node sends its own position to the Sink node and completes the optimization operation by the Sink node. Each group of sensor nodes can be expressed as:

$$
W=\left(w_{1}, w_{2}, \ldots, w_{i}, w_{N}\right)
$$

The distance between the node $w_{i}$ and the receiving point $\left(a_{k}, b_{k}\right)$ is expressed as:

$$
d_{i, k}=\sqrt{\left(x_{i}-a_{k}\right)^{2}+\left(y_{i}-b_{k}\right)^{2}}
$$

The probability of receiving point $\left(a_{k}, b_{k}\right)$ to the node $w_{i}$ signal is expressed as:

$$
P_{i, k}= \begin{cases}1 & d_{i, k} \leq R \\ 0 & d_{i, k}>R\end{cases}
$$

The probability that the receiver $\left(a_{k}, b_{k}\right)$ can receive the number one sent by this group of nodes is expressed as:

$$
P\left(a_{k}, b_{k}\right)=1-\prod\left(1-P_{i, k}\right)
$$

Finally, the signal coverage rate of the transmitter $W$ to the region is expressed as:

$$
G_{W}=\frac{\sum P\left(a_{k}-b_{k}\right)}{a^{*} b}
$$

\subsection{Optimization of wireless sensor network coverage for mobile nodes}

The research of wireless sensor network coverage optimization is a hot topic at present. Through the study of the coverage optimization, the network coverage, energy consumption and network life and stability can be improved effectively. At present, the commonly used algorithms include virtual force algorithm (VFA), particle 
swarm optimization (PSO), frog leaping algorithm (SFLA) and firefly algorithm (FA). However, these algorithms have some drawbacks in wireless sensor network coverage optimization. They are not well integrated with coverage optimization models. For example, the structure of particle swarm optimization (PSO) is too complex, which results in too slow computing speed and too poor real-time performance. Frog jumping algorithm performance is poor. The final coverage is not satisfactory. It does not meet the user's service requirements. Some algorithms have many parameters, leading to the network model is too complicated. In response to these problems, CSFOA is used to avoid these drawbacks. It effectively solves the wireless sensor network coverage optimization problem of mobile nodes. The flow chart of the coverage algorithm of the mobile node based on CSFOA is shown in Figure 5.

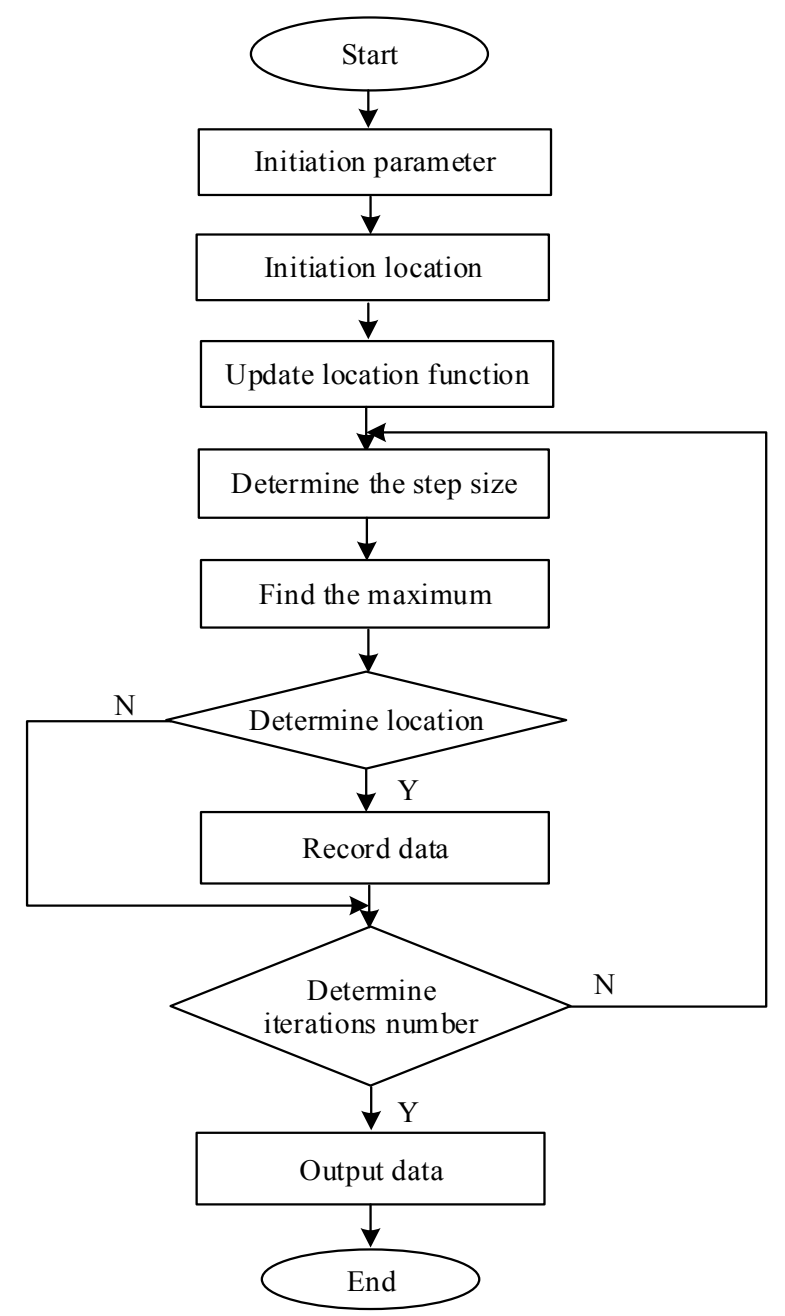

Fig. 5. The flow chart of the coverage algorithm of the mobile node based on CSFOA 
In order to verify the superiority of CSFOA in the coverage optimization of wireless sensor networks in mobile nodes, under the condition that the number of iterations is 200 , the coverage area is $50 m * 50 m$ and the nominal effective radius of node is $5 m$, five algorithms, namely, CSFOA, improved virtual force particle swarm optimization (VFPSO), improved bat algorithm (BA), improved chaos fish algorithm and DS-FOA, are used to simulate wireless sensor network coverage optimization. The average of the results of 50 experiments is taken as the final result. The final results are shown in Table 1 (set the theoretical coverage is 1 ).

Table 1. Comparison of algorithm coverage

\begin{tabular}{|l|c|}
\hline \multicolumn{1}{|c|}{ Coverage algorithm } & Coverage rate (\%) \\
\hline CSFOA & 98.24 \\
\hline DS-FOA & 95.54 \\
\hline Improved VFPSO & 98.01 \\
\hline Improved BA & 89.26 \\
\hline Improved fish swarm algorithm & 96.3 \\
\hline
\end{tabular}

As can be seen from Table 1, under the same circumstances, compared with other improved algorithms, the CSFOA has the highest network coverage, and the theoretical coverage is $98.24 \%$. Compared with DS-FOA, improved VFPSO, improved BA and improved fish algorithm, the CSFOA algorithm increased by $2.7 \%, 0.23 \%, 8.98 \%$ and $1.94 \%$ respectively. Because CSFOA is closely integrated with the high performance of wireless sensor network model and algorithm, it has better effect on the coverage optimization of wireless sensor networks in mobile nodes.

Through the data display and analysis of simulation experiments, it can be seen that CSFOA has good performance (convergence accuracy and convergence speed) in the coverage optimization of wireless sensor networks for mobile nodes. Compared with other improved intelligent optimization algorithms, the proposed algorithm can better integrate the network model. It has better results and the process is more stable. The network coverage is higher. Therefore, it is more suitable for mobile node wireless sensor network coverage optimization.

\subsection{Optimization of wireless sensor network coverage for fixed position nodes}

In addition to the model of the mobile node described in the previous section, the wireless sensor network coverage model also has a fixed position node model. At present, many scholars have done a thorough research on the problem of wireless sensor network coverage optimization for fixed position nodes. In a wireless sensor network model with fixed location nodes, a large number of sensor nodes are randomly deployed in the monitoring area. After deployment, the location of nodes will not change. There are many problems in this model. For example, a large number of nodes are redundant, resulting in low utilization of nodes and irregular coverage of nodes. The coverage is not enough. The energy consumption of the nodes is fixed, which leads to the high energy consumption of the whole network and so on. From 
the current research, redundant nodes in the coverage model are eliminated by grid coverage and coverage radius nodes. However, the traditional algorithm has such problems as high complexity or low convergence accuracy. It cannot achieve ideal results in coverage optimization of wireless sensor networks with fixed location nodes. In view of the above problems, the CSFOA algorithm is used to solve the dynamic radius planning of fixed position nodes, and the optimization of network coverage is realized. The simulation experiment shows the superiority and effectiveness of the CSFOA algorithm in the wireless sensor network coverage optimization of fixed position nodes.

Figure 6 is a comparison of two improved Drosophila algorithms for DS-FOA and CSFOA in wireless sensor network coverage optimization at fixed location nodes. It can be seen that after 500 iterations, two algorithms achieve high accuracy, and two algorithms have fast convergence speed. Compared with DS-FOA, CSFOA can better combine the wireless sensor network model with fixed position nodes. It has better optimization performance. In the first 50 iterations, CSFOA can quickly achieve high precision convergence. In the 50500-iterative process, the algorithm can jump out of the local optimal by self-correction, and further improve the accuracy and achieve better results. After the 500 iterations, the result of CSFOA is much more accurate than that of DS-FOA. The two kinds of fruit fly algorithms are compared as shown in Figure 6.

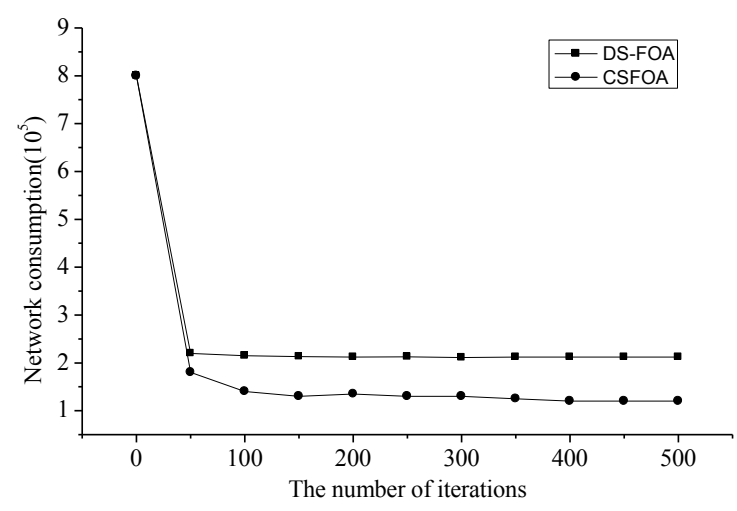

Fig. 6. Comparison of two kinds of fruit fly algorithms

By analyzing the data of the simulation experiment, CSFOA also has good performance on the wireless sensor network coverage optimization problem of fixed position nodes. While guaranteeing the coverage of wireless sensor networks, the energy consumption of the network can be greatly reduced. Compared with other improved intelligent optimization algorithms for coverage optimization problems in fixed location nodes, this algorithm can better integrate network models. It has a better solution and a simpler solution process. Finally, it gets high network coverage (generally, it is $100 \%)$. A large number of redundant sensor nodes have been eliminated and the energy consumption of the network is reduced. It is more suitable for wireless sensor network coverage optimization of fixed position nodes. 


\section{Conclusions}

Based on a new type of intelligent algorithm, the change step of fruit fly optimization algorithm (CSFOA) is proposed. At the same time, the mathematical modeling of two network models is carried out respectively. Finally, combined with the corresponding network model, the improved algorithm is applied to wireless sensor network. The results show that the improved algorithm is effective and superior to the coverage optimization of wireless sensor networks. The specific performance is as follows:

First, the improved fruit fly algorithm is combined with two coverage models of wireless sensor networks. Through the comparison test, it is proved that the solution of this paper is better than the previous solution.

Second, based on the foraging behavior of fruit flies, the fruit fly optimization algorithm was proposed. Fruit flies have a sensitive sense of smell and vision. It can quickly and accurately find the food location. Therefore, the FOA algorithm has the characteristics of less parameters, faster convergence rate and higher accuracy.

Third, the fruit fly optimization algorithm has many advantages, there are some obvious drawbacks. For example, the algorithm tends to fall into the local optimal convergence. The step size of the algorithm will have a great influence on the optimization efficiency and the result.

Finally, based on the Drosophila algorithm, the change step of fruit fly optimization algorithm (CSFOA) is proposed. At the same time, CSFOA algorithm is applied to the coverage optimization problem of wireless sensor network. Through a series of simulation experiments, the effectiveness and superiority of CSFOA on the coverage optimization of wireless sensor networks is verified. The optimization of wireless sensor network coverage is realized.

\section{Acknowledgement}

This paper is supportted by Project of the Education Department of Jilin Province, "13th Five-Year" science and technology project "in finance and Economics Colleges Construction of cross platform integrated simulation training" (contract number: JJKH20170122KJ).

\section{$7 \quad$ References}

[1] Cheng, C. T., Chi, K. T., \& Lau, F. C. M. A delay-aware data collection network structure for wireless sensor networks. IEEE Sensors Journal, 2017, vol. 11(3), pp. 699-710. https://doi.org/10.1109/JSEN.2010.2063020

[2] Sun, Y., Zhang, S., Xu, H., \& Lin, S. Cooperative communications for wireless ad hoc and sensor networks in 2013. International Journal of Distributed Sensor Networks, 2014, vol. 1, pp. 155-184. https://doi.org/10.1155/2014/274378 
Paper-Wireless Sensor Network Coverage Optimization Based on Fruit Fly Algorithm

[3] Li, M., \& Lin, H. J. Design and implementation of smart home control systems based on wireless sensor networks and power line communications. IEEE Transactions on Industrial Electronics, 2015, vol. 62(7), pp. 4430-4442. https://doi.org/10.1109/TIE.2014.2379586

[4] Luo, J., Hu, J., Wu, D., \& Li, R. Opportunistic routing algorithm for relay node selection in wireless sensor networks. IEEE Transactions on Industrial Informatics, 2017, vol. 11(1), pp. 112-121. https://doi.org/10.1109/TII.2014.2374071

[5] Rezvani, M., Ignjatovic, A., Bertino, E., \& Jha, S. Secure data aggregation technique for wireless sensor networks in the presence of collusion attacks. Dependable \& Secure Computing IEEE Transactions on, 2015, vol. 12(1), pp. 98-110. https://doi.org/10.1109/TDSC. 2014.2316816

[6] Cheng, C. T., Chi, K. T., \& Lau, F. C. M. A delay-aware data collection network structure for wireless sensor networks. IEEE Sensors Journal, 2017, vol. 11(3), pp. 699-710. https://doi.org/10.1109/JSEN.2010.2063020

[7] Ruela, A. S., Cabral, R. D. S., Aquino, A. L. L., \& Guimarães, F. G. Memetic and evolutionary design of wireless sensor networks based on complex network characteristics, 2017, vol. 1(2), pp. 33-53.

[8] Younis, O., Krunz, M., \& Ramasubramanian, S. Node clustering in wireless sensor networks: recent developments and deployment challenges. Network IEEE, 2015, vol. 20(3), pp. 20-25. https://doi.org/10.1109/MNET.2006.1637928

[9] Yao, Y., Cao, Q., \& Vasilakos, A. V. Edal: an energy-efficient, delay-aware, and lifetimebalancing data collection protocol for wireless sensor networks. IEEE/ACM Transactions on Networking, 2015, vol. 23(3), pp. 810-823. https://doi.org/10.1109/TNET.2014.230659 2

[10] Climent, S., Sanchez, A., Blanc, S., Capella, J. V., \& Ors, R. Wireless sensor network with energy harvesting: modeling and simulation based on a practical architecture using real radiation levels. Concurrency \& Computation Practice \& Experience, 2016, vol. 28(6), pp. 1812-1830. https://doi.org/10.1002/cpe.3151

\section{Authors}

Ren Song, Zhichao Xu, and Yang Liu are with Jilin University of Finance and Economics, Jilin, China.

Article submitted 12 January 2018. Resubmitted 23 February 2018. Final acceptance 15 March 2018. Final version published as submitted by the authors. 